\title{
New Freeform Manufacturing Chains based on atmospheric Plasma Jet Machining
}

\author{
T. Arnold \\ thomas.arnold@iom-leipzig.de
}

\section{G. Boehm}

H. Paetzelt
Leibniz Institute of Surface Modification, Permoserstr. 15, 04318 Leipzig, Germany

Leibniz Institute of Surface Modification, Permoserstr. 15, 04318 Leipzig, Germany

New manufacturing chains for precise fabrication of asphere and freeform optical surfaces including atmospheric Plasma Jet Machining (PJM) technology will be presented. PJM is based on deterministic plasma-assisted material removal. It has the potential for flexible and cost-efficient shape generation and correction of small and medium-sized optical freeform elements. The paper discusses the interactions between the plasma tools and optical fused silica samples in the context of the pre-machined and intermediate surface states and identifies several plasma jet machining methods for freeform generation, surface correction, and finishing as well as suitable auxiliary polishing methods. The successful application of either processing chain is demonstrated.

[DOI: http://dx.doi.org/10.2971/jeos.2016.16002]

Keywords: Freeform manufacturing, aspheres, plasma jet machining, plasma jet polishing

\section{INTRODUCTION}

Currently, an increasing demand for individual and complex shaped optical elements like non-standard aspheres, acylinders, or freeform elements is observed. Especially, the use of freeform elements in optical systems provides new functionalities in illumination and imaging applications and yields less optical surfaces and thus more compact system designs. Recent advances in manufacturing technologies give more and more freedom to optical system designers. However, once the machinability of complex formed surfaces has been proven the tolerances are getting more and more stringent, particularly for scientific equipment, e.g. space and earth-based spectrometers or telescopes, or laser beam shaping applications, high precision of optical freeform surfaces is required.

Classical manufacturing of such optics generally involves intense sub-aperture polishing after the freeform grinding process to generate a specular surface and to remove sub-surface damage (SSD). Depending on the grinding process material removal of $15-30 \mu \mathrm{m}$ is necessary to get good optical surface quality in the high spatial frequency range. Furthermore, subaperture polishing often leads to significant shape alteration, particularly in the case of challenging surface shapes showing significant variations of local surface curvature, which originates from the corresponding local variation of polishing removal rates. After the polishing process the surface roughness is usually within the specified range, though an unintended shape deviation of 1-2 $\mu$ m often occurs. Thus, the subsequent application of deterministic surface error correction methods is required to reach the specifications, which employ costintensive and time-consuming sub-aperture techniques like
Computer Controlled Polishing (CCP), Magneto Rheological Finishing (MRF), or Ion Beam Figuring (IBF).

In the paper we introduce alternative approaches to optical freeform element manufacturing based on atmospheric Plasma Jet Machining and utilizing a shape-preserving postpolishing process. Two different process chains are discussed where one of it comprises plasma based methods only. The process chains are mainly dedicated to the fabrication of precise small and medium-sized optics with diameters of 5-150 $\mathrm{mm}$ that come as single pieces or small batches. However, the technique has the potential to be scaled up to larger work pieces and unit numbers.

\section{PLASMA JET MACHINING}

Plasma Jet Machining (PJM) employs atmospheric reactive plasma interacting with the surface [1]. The plasma source consists mainly of a coaxial electrode system. The inner electrode is made of coaxially arranged tubes that terminate in a nozzle. The electrode system guides high frequency power (either RF at $13.56 \mathrm{MHz}$ or microwave at $2.45 \mathrm{GHz}$ ) from the high frequency generator to the nozzle (see Figure 1). At the nozzle tip high electric field strength leads to ionization of the gas fed through the tubes and form a jet-like gas discharge. Optional apertures and nozzles can be attached to scale and stabilize the plasma jet flow. Working gases are argon and helium as well as fluorine containing gases like $\mathrm{SF}_{6}$ or $\mathrm{CF}_{4}$ that are dissociated to form free highly reactive fluorine atoms and other radicals. 


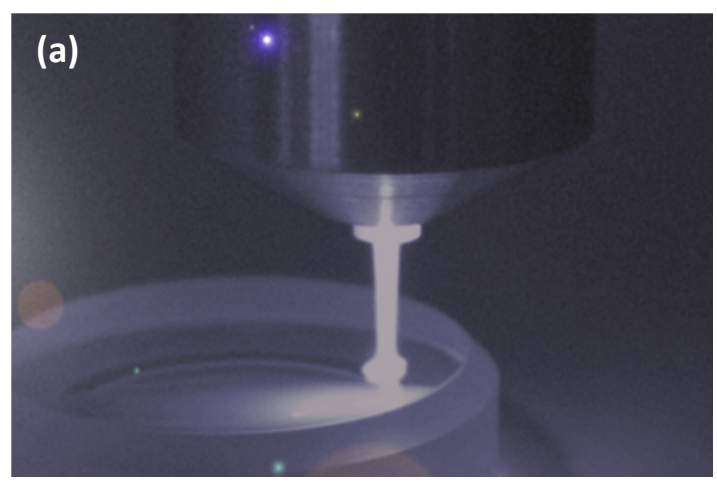

(b)

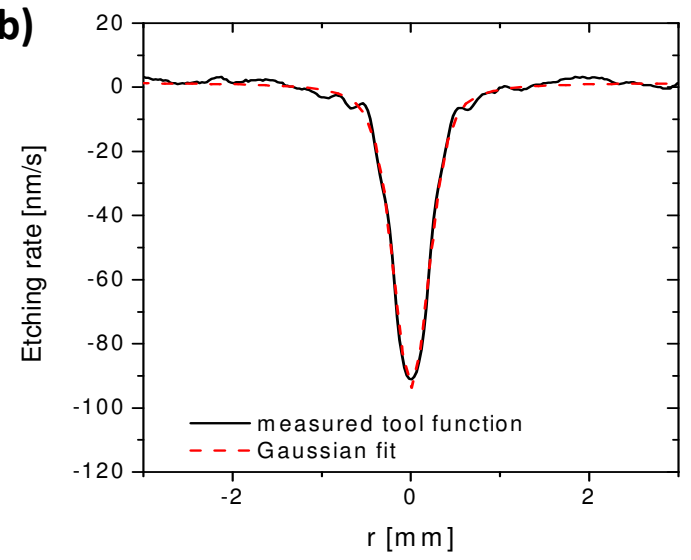

FIG. 1 (a) Atmospheric reactive plasma jet on a fused silica optic, (b) Typical tool function.

The reactive species undergo chemical reactions with surface atoms where volatile compounds are created. In this way a material removal is achieved. Hence, PJM technology works best on pure silicon based materials like fused silica, silicon, silicon carbide, or ULE. Silicon, oxygen, titanium and carbon form gaseous fluorinated or oxidized compounds as the main reaction products. Other gases like oxygen and nitrogen can be added to the plasma as enhancer of fluorine formation or for shielding the plasma against the surrounding environment, respectively.

The power consumption of the plasma source ranges from $2 \mathrm{~W}$ to $400 \mathrm{~W}$ depending on the total gas flow, which can be adjusted between 200 and 3000 sccm (standard cubic centimeters per minute). The plasma jet acts as a local tool revealing a nearly rotationally symmetric Gaussian tool function. The full width at half maximum of the Gaussian varies typically between $0.3 \mathrm{~mm}$ and $8 \mathrm{~mm}$ depending on the setup. Several plasma sources producing different sizes of tool functions have been developed. By utilizing high-rate PJM sources material removal rates of some $10 \mathrm{~mm}^{3} / \mathrm{min}$ can be achieved while low-rate sources produce small spatially confined jets and material removal rates of $0.01 \mathrm{~mm}^{3} / \mathrm{min}$ or less. In order to perform a deterministic machining process either the plasma source or workpiece is moved by a computercontrolled motion system comprising 3 or 5 motion axes to feed the plasma jet over the surface. The motion scheme is determined by local dwell times calculated by sophisticated numerical deconvolution algorithms. These algorithms are capable to take also into account special dynamic characteristics of the tool function during the machining process, e.g. changes in etching rate due to surface heating effects [2].
Consequently, there are two plasma based machining processes available: one which is suitable for the generation of freeform shapes, that finds its classical equivalent in mechanical grinding; the other is a fine correction step comparable to ion beam figuring (IBF) or magneto-rheological finishing (MRF).

The major advantage of PJM over mechanical-abrasive correction methods is the atomistic material removal mechanism without exerting any significant mechanical forces onto the surface. Hence, the tool does not introduce sub-surface damages. The plasma jet forms a long-term stable tool predestinated for freeform fabrication. In the following we discuss the application of PJM to fused silica surfaces.

\section{PJM ETCHING CHARACTERISTICS}

In order to benefit from the dry etching plasma based figuring process it is important to be able to preserve the surface quality that has been obtained by mechanical pre-machining. Alternatively, the method should be capable to even improve the roughness. It is well known from many investigations of HF wet etching or reactive plasma etching of fused silica using highly reactive species that a characteristic surface roughness evolves [3, 4]. High spatial frequency structures occur in strong dependence on initial surface roughness, chemical contaminants, or sub-surface damage (SSD), since fluorine atom attack on the surface is highly selective. Also, etching depth has a significant influence on the final roughness. Therefore roughness evolution during plasma treatment has been investigated on differently prepared fused silica samples. Figure 2 illustrates the surface state after plasma etching of ground and polished surfaces.

In Figure 2(a) a coarse ground and subsequently polished surface has been plasma treated. Polishing grade was P2 according to the ISO 10110 nomenclature. Etching depth was only approximately $10 \mu \mathrm{m}$. The microscope image reveals typical deep structures originating from hidden micro-cracks produced by the grinding process. In Figure 2(b) a surface with standard polishing grade P3 has been plasma etched. Here, etching depth was approximately $200 \mu \mathrm{m}$. The root mean square roughness of the surface, denoted by $S q$, increased from $S q=2 \mathrm{~nm}$ to $S q=100-150 \mathrm{~nm}$. Obviously, the P3 polishing removed the SSD to some extent. In Figure 2(c) a sample is shown that has been extensively polished to grade P4. After plasma etching of approximately $200 \mu \mathrm{m}$ the roughness Sq is still in the range $2-3 \mathrm{~nm}$, revealing that the SSD was already at a low level after polishing. These examples clearly indicate the influence of grinding and polishing and especially the SSD induced by those methods to the roughness evolution during plasma jet etching. Thus, it is of great importance to prepare a fused silica surface in a proper way in order to apply plasma based machining processes subsequently. For planar or spherical samples this is mostly affordable due to the use of fullaperture tools. However, to make asphere or freeform surfaces ready for plasma etching (e.g. for figure error correction) requires extensive sub-aperture polishing after grinding inducing significant degradation of surface figure, which increases the correction effort. 


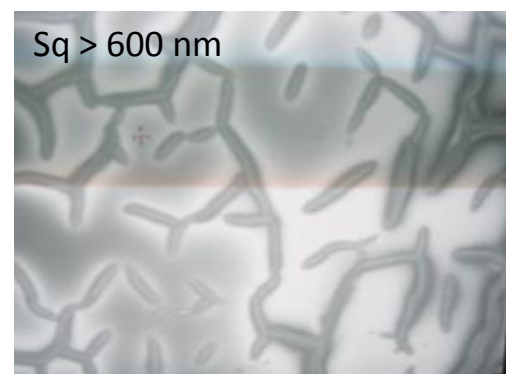

(a)

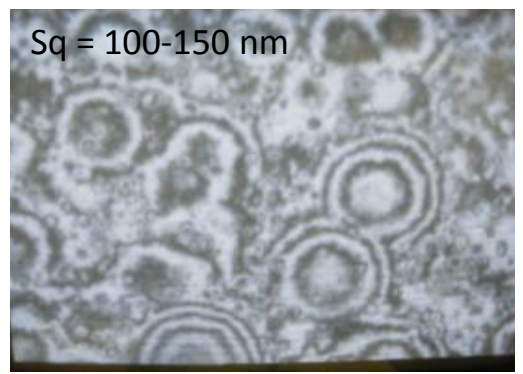

(b)

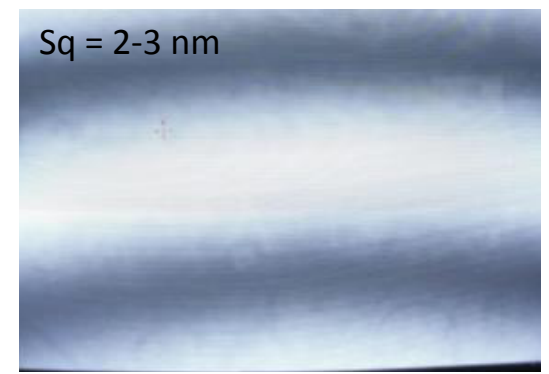

(c)

FIG. 2 Ground and polished fused silica surfaces after plasma jet etching: (a) coarse ground and polished to grade $\mathrm{P}_{2}$, (b) ground and polished to grade $\mathrm{P}_{3}$, (c) ground and polished to grade $\mathrm{P}_{4}$.

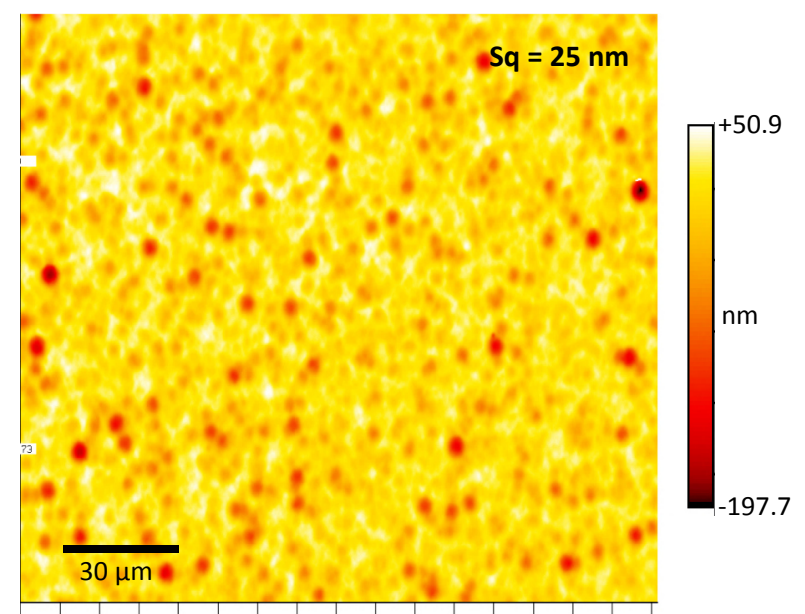

(a)

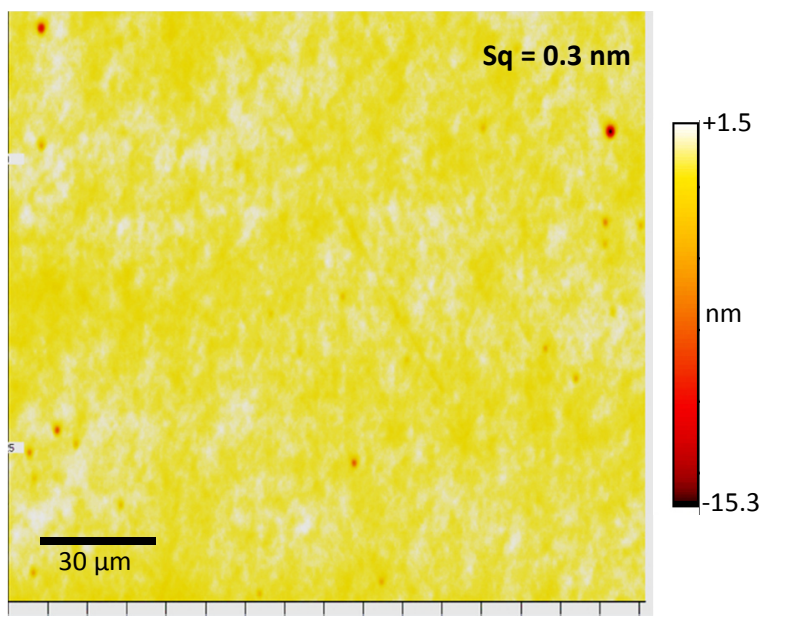

(b)

FIG. 3 (a) Mechanically polished $P_{3}$ surface after plasma jet etching, (b) plasma jet polished surface after plasma jet etching.

We therefore propose a plasma jet process for shape preserving polishing or subsurface damage removal, respectively. It has been shown that specially designed inert gas plasma jets can also be used for polishing of rough fused silica surfaces. For that purpose an inert gas plasma jet without any reactive species is employed forming a high temperature spot on the surface. As a result the viscosity of the glass on the surface is decreased and surface tension and capillary forces lead to redistribution of material. The surface smoothing mechanisms are supposed to be similar to those occurring in flame polishing or laser polishing [5]. Figure 3(a) shows an interference microscope image of a mechanically polished surface of quality P3, which has been plasma treated to etch $30 \mu \mathrm{m}$. The occurring defects indicate the presence of SSD causing a final roughness of $25 \mathrm{~nm}$ RMS.

In contrast to this a final roughness of $<0.5 \mathrm{~nm}$ is obtained when a thermal plasma polishing step is applied prior to the etching step on the same surface (see Figure 3(b). This is at a sufficient level for many optical applications. From the findings it can be concluded that plasma jet polishing heals microcracks buried in the near-surface region located below the Beilby layer [4].

A representative plasma jet polishing result is shown in Figure 4 . One half of a fine ground aspheric surface with roughness $\mathrm{Sq} \sim 250 \mathrm{~nm}$ has been treated by the plasma jet, yielding a significantly decreased roughness of $S q$ of $0.2-0.3 \mathrm{~nm}$. Roughness has been determined using interference microscopy. AFM measurements have proven this value. Consequently, roughness is decreased leaving finally an optically smooth surface.

\section{MANUFACTURING CHAINS}

With the set of three plasma jet based methods for surface polishing, figuring, and correction it is possible to define processing chains for the manufacturing of aspheres or freeform elements utilizing plasma jet methods only. Additionally, PJM can be appropriately combined with mechanical sub-aperture bonnet polishing, or special soft tool polishing that is capable to maintain the freeform surface figure and to improve the micro-roughness further.

A very challenging task in freeform manufacturing is the generation of refractive laser beam shaping elements. Depending on the optical setup these elements have very different designs and dimensions but most of them can be manufactured simply out of planar optical windows or of spherical lenses. The plasma-based manufacturing chain then ideally starts from a best fit standard optical element and the goal shape is machined by high-rate PJM tools. The manufacturing chain (A) comprises the following steps:

(i) Plasma jet polishing: Depending on the polishing grade and SSD level found on the surface this is performed prior to plasma jet etching. However, plasma polishing rate lies in the range of $1 \mathrm{~cm}^{2} / \mathrm{min}$, which is rather slow. 


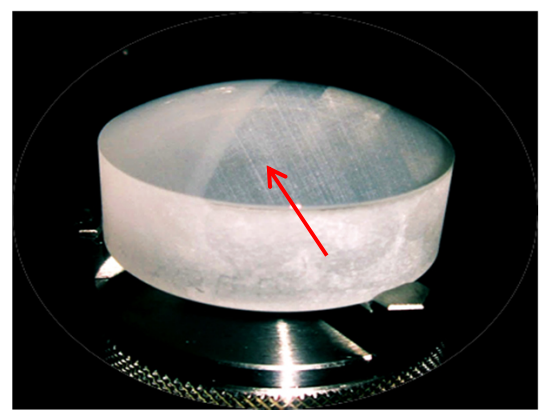

(a)

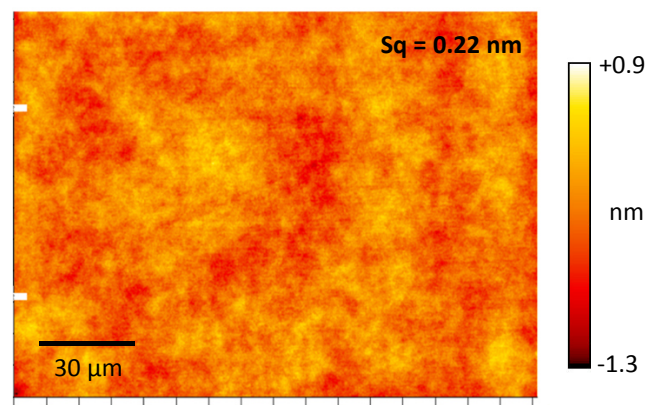

(b)

FIG. 4 (a) Fine ground fused silica asphere that has been plasma jet polished on the right-hand side (indicated by the red arrow), (b) Corresponding roughness measurement of the polished surface.

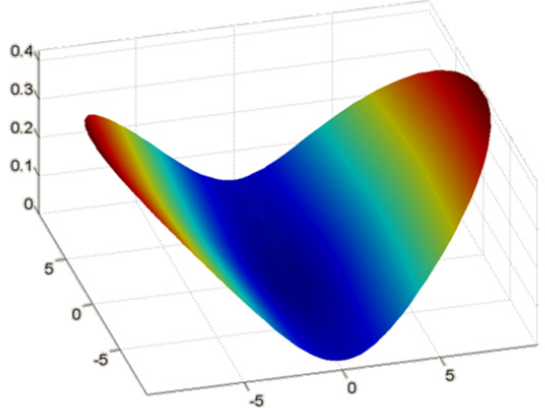

(a)

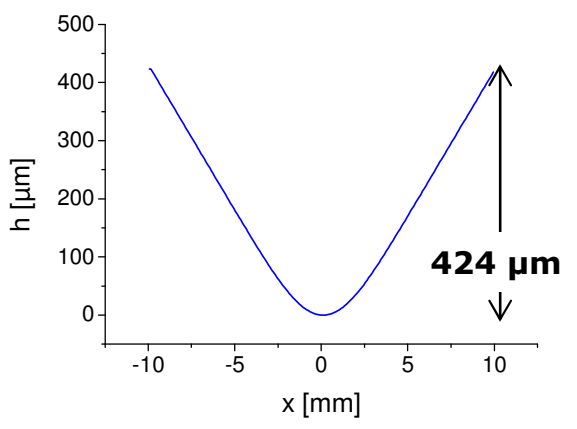

(b)

FIG. 5 (a) Design of a freeform optical element for laser beam shaping. (b) Cross section of the freeform shape.

(ii) High-rate PJM: Removal depths of 1-1000 $\mu \mathrm{m}$ can be realized to generate an asphere or freeform. The employed PJM tool width (FWHM) is mainly determined by the gradients and curvatures of the theoretical shape prescription and varies typically between $2.5 \mathrm{~mm}$ and $4.5 \mathrm{~mm}$. The residual figure error is typically less than $2 \%$ of the PV of the targeted shape. As discussed before, the surface roughness occurring during this figuring step depends strongly on the amount of removal as well as on the mechanical pre-treatment (grinding, polishing), cleaning of the initial surface, and plasma polishing. In case of proper preparation at least one order of magnitude lower micro-roughness compared to conventional grinding processes has been achieved also for high removal depths of some $100 \mu \mathrm{m}$. Typically a roughness $S q<50 \mathrm{~nm}$ is achieved, which enables interferometric measurements.

(iii) Sub-aperture bonnet polishing: Since no SSD is induced during PJM only very low material removal of typically 1-2 $\mu \mathrm{m}$ by sub-aperture bonnet polishing is needed afterwards to reduce roughness to typically $S q<5 \mathrm{~nm}$. Therefore, surface figure changes only within a few $100 \mathrm{~nm}$ PV and the formation of mid-spatial structures is minimized.

(iv) PJM fine correction: This step is characterized by a relatively low material removal of a few micrometer at maximum to achieve residual figure errors of $<100 \mathrm{~nm} \mathrm{PV}$. Roughness will more or less not be altered.

(v) Soft tool polishing: Very soft polishing tools can be applied finally to reach optical surface quality with a micro-roughness of $S q<1 \mathrm{~nm}$ after removal of only a few $100 \mathrm{~nm}$ leaving the surface figure more or less unchanged.

As an example the fabrication of laser beam shaping elements is discussed in detail. The example given in Figure 5 shows a freeform element with about $400 \mu \mathrm{m}$ PV deformation that has been generated on a fused silica optical window. Since the substrate was already well-polished, a plasma jet polishing step could be omitted. Thus, the first process step was shape generation using a tool with FWHM of approximately $3 \mathrm{~mm}$ and material removal rate of approximately $2 \mathrm{~mm}^{3} / \mathrm{min}$. The machining time of this step was approximately 40 minutes leaving a figure error of 4-6 $\mu \mathrm{m}$ PV (see Figure 6(a)). The surface micro-roughness measured by interference microscopy has been determined to be less than $10 \mathrm{~nm}$.

This value has also been achieved in the region of the largest removal. In order to decrease roughness bonnet polishing has been applied. Figure 6(b) shows an interference microscope image of the polished surface. Roughness value has been determined to be $S q=2 \mathrm{~nm}$. Though, a distinct pattern becomes visible which is due to slight runout of the tool. For the subsequent correction step a smaller plasma jet tool with FWHM of $0.8 \mathrm{~mm}$ and a material removal rate of $0.003 \mathrm{~mm}^{3} / \mathrm{min}$ has been used to reduce the figure error $0.9 \mu \mathrm{m}$ PV or $0.2 \mu \mathrm{m}$ RMS. (Figure 7(a)). After PJM treatment the surface has been polished by the soft polishing tool maintaining the shape within less than $100 \mathrm{~nm}$ PV. The measurement shown in Figure 7(b) reveals a micro-roughness of $S q=0.6 \mathrm{~nm}$.

An alternative manufacturing chain (B) comprises only plasma jet methods while omitting any mechanical polishing. 


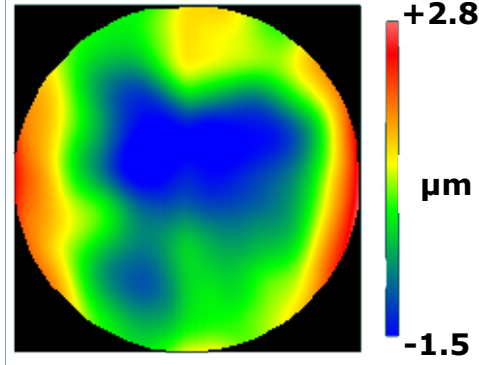

(a)

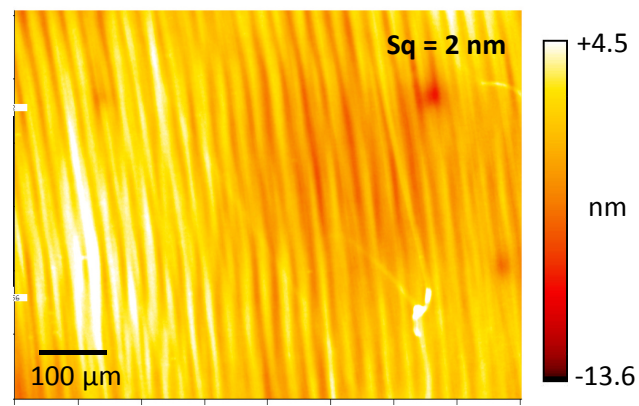

(b)

FIG. 6 (a) Interferometric measurement of residual figure error after high-rate plasma jet etching and sub-aperture bonnet polishing (b) Interference microscope image (10x) of bonnet-polished fused silica surface. The periodic structure is most probably due to run-out of the polishing tool.

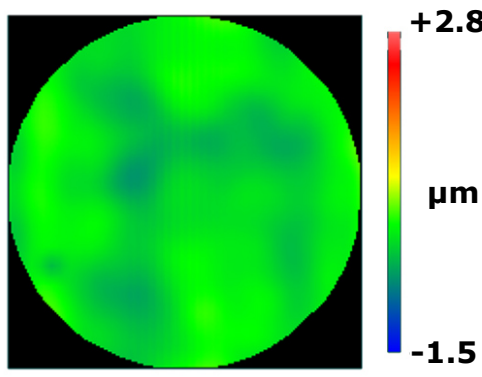

(a)

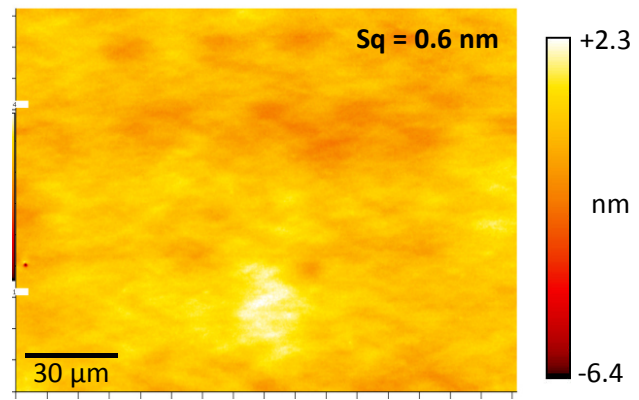

(b)

FIG. 7 (a) Residual figure error after plasma jet fine correction (PV $0.9 \mu \mathrm{m}$ PV, RMS $0.2 \mathrm{~nm}$ ). (b) Interference microscope image (50x) of soft tool polished surface.

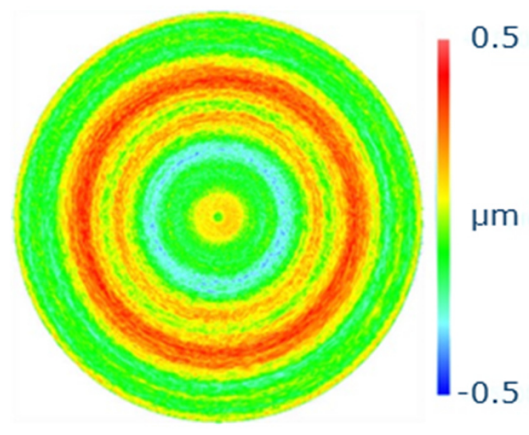

(a) PV $0.89 \mu \mathrm{m}$, RMS $0.15 \mu \mathrm{m}$

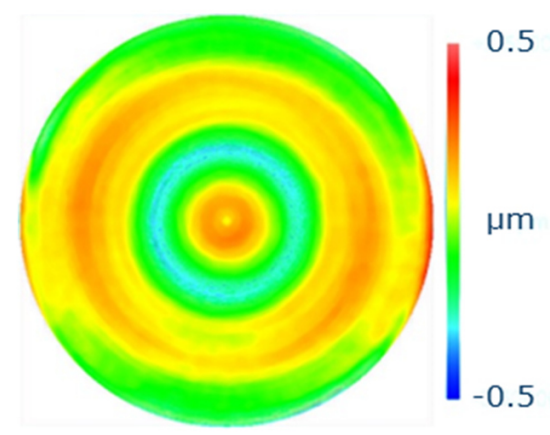

(b) $\mathrm{PV} 0.72 \mu \mathrm{m}$, RMS $0.13 \mu \mathrm{m}$

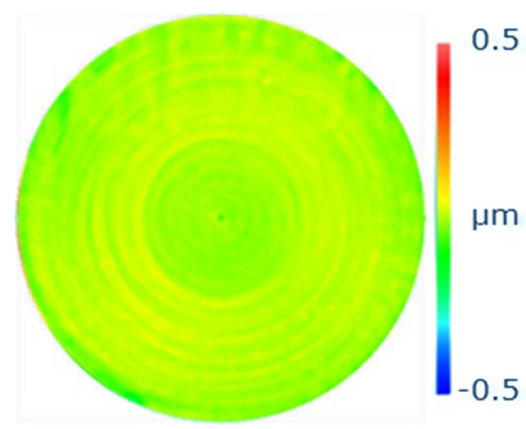

(c) PV $0.190 \mu \mathrm{m}$, RMS $0.015 \mu \mathrm{m}$

FIG. 8 Residual figure errors (a) of ground asphere, (b) after plasma jet polishing, (c) after plasma jet fine correction.

In this case the asphere or freeform shape is generated by a (1) freeform grinding process ending up with a fine ground surface quality with roughness $S q \sim 250 \mathrm{~nm}$ and a figure error of typically less than $2 \mu \mathrm{m}$ PV. The surface is then treated with (2) plasma jet polishing, where the roughness is decreased to a value of $S q<1 \mathrm{~nm}$ and SSD is removed while the figure error remains more or less unchanged. Finally a (3) plasma jet fine correction step is employed to diminish the figure error and keeping the roughness.

An example for the successful application of this manufacturing chain is given in Figure 8. A fine ground convex asphere (diameter $50 \mathrm{~mm}$, radius of curvature) with surface figure error of $0.89 \mu \mathrm{m}$ PV has been plasma jet polished. The polishing process lasted approximately 45 minutes. Form measurements using a LuphoScan profiler indicate that the surface shape has barely altered from its initial value. Microroughness has been determined to be $S q=0.25 \mathrm{~nm} \pm 0.05 \mathrm{~nm}$ over the entire surface. After approximately $30 \mathrm{~min}$ of plasma jet fine correction the shape has been significantly improved.

Depending on the specific task and surface geometry either chain (A) or (B) is more appropriate with regard to overall machining time and process convergence. Today, ultra-precision grinding of rotationally symmetric aspheres is a state-of-theart technology. However, if ultra-high form accuracy $<20 \mathrm{~nm}$ RMS and high quality surface (scratch-dig $<20-10)$ is required, tremendous effort is necessary to reach the specifications by utilizing conventional processin chains including several iteration cycles of sub-aperture polishing and correction processes like MRF or IBF. Employing chain (B) in those cases can significantly save machining time since only two steps need to be applied. The main advantage is the avoidance of abrasive slurries, elaborate workpiece cleaning between machining and measurement steps, or the utilization of expensive and slow IBF processes. Thus, chain (B) has the potential 
to greatly increase the manufacturing efficiency as far as the preform can be manufactured by precision freeform grinding. For optical elements that exhibit complex surfaces such as aspheres with inflection or turning points, or freeforms, form generation by precision grinding might be an intricate problem. In order to achieve the required accuracy, in many cases it can be overcome by plasma jet machining according chain (A). Although several steps must be applied including slurrybased polishing, chain (A) appears to be more effective and straight-forward regarding form accuracy than conventional processes due to the flexibility with respect to form and surface gradients.

\section{CONCLUSION}

In the paper the characteristics of PJM have been discussed and two processing chains for asphere and freeform manufacturing have been introduced that comprise plasma jet polishing, plasma jet high-rate figuring and correction, as well as auxiliary sub-aperture polishing steps. Both chains provide the opportunity for precise fabrication of optical elements with difficult surface shape like non-standard aspheres, acylinders or freeforms. In contrast to the conventional grinding and polishing techniques the non-contact PJM technology has nearly no geometrical limits. Furthermore, due to the pure chemical removal mechanism no sub-surface damage is induced. Therefore, the amount of polishing removal after PJM is reduced to a few microns and hence, shape alteration during the mechanical polishing process is minimized. Employing the second process chain purely based on plasma methods allows omitting any mechanical sub-aperture polishing avoiding the problems and challenges connected therewith.

\section{ACKN OWLEDGEMENTS}

The authors gratefully acknowledge financial support within the framework of the InnoProfile Transfer project funded by German Ministry of Education and Research (BMBF) under contract no. 03IPT706X.

\section{References}

[1] T. Arnold, G. Böhm, H. Paetzelt, "Ultra-precision surface machining with reactive plasma jets," Contrib. Plasma Phys. 54, 145-154 (2014).

[2] J. Meister, and T. Arnold, "New process simulation procedure for high-rate Plasma Jet Machining," Plasma Chem. Plasma Process $31,91-107$ (2011).

[3] Q. Xin, N. Li, J. Wang, B. Wang, G. Li, F. Ding, and H. Jin, "Surface roughening of ground fused silica processed by atmospheric inductively coupled plasma," Appl. Surf. Sci. 341, 142-148 (2015).

[4] Z. Zheng, X. Zu, X. Jiang, X. Xiang, J. Huang, X. Zhou, C. Li, et al., "Effect of HF etching on the surface quality and laser-induced damage of fused silica," Opt. Laser Technol. 44, 1039-1042 (2012).

[5] P. Cormont, P. Combis, L. Gallais, C. Hecquet, L. Lamaignère, and J. L. Rullier, "Removal of scratches on fused silica optics by using a $\mathrm{CO}_{2}$ laser," Opt. Express 21, 28272-282289 (2013). 